\title{
Optimal Discrete-Valued Control Computation
}

\author{
Changjun Yu • Bin Li • Ryan Loxton • \\ Kok Lay Teo
}

Received: date / Accepted: date

\begin{abstract}
In this paper, we consider an optimal control problem in which the control takes values from a discrete set and the state and control are subject to continuous inequality constraints. By introducing auxiliary controls and applying a time-scaling transformation, we transform this optimal control problem into an equivalent problem subject to additional linear and quadratic constraints. The feasible region defined by these additional constraints is disconnected, and thus standard optimization methods struggle to handle these constraints. We introduce a novel exact penalty function to penalize constraint violations, and then append this penalty function to the objective. This leads to an approximate optimal control problem that can be solved using standard software packages such as MISER. Convergence results show that when the penalty parameter is sufficiently large, any local solution of the approximate problem is also a local solution of the original problem. We conclude the paper with some numerical results for two difficult train control problems.
\end{abstract}

\author{
Changjun $\mathrm{Yu}$ \\ Shanghai University, SHANGHAI, CHINA \\ Curtin University, Perth, WA, AUSTRALIA \\ E-mail: yuchangjun@126.com \\ Bin Li \\ Harbin Institute of Technology, Harbin, CHINA \\ E-mail: abissing@163.com \\ Ryan Loxton \\ Curtin University, Perth, WA, AUSTRALIA \\ E-mail: r.loxton@curtin.edu.au \\ Kok Lay Teo \\ Curtin University, Perth, WA, AUSTRALIA \\ E-mail: k.l.teo@curtin.edu.au
}




\section{Introduction}

In many practical optimal control problems arising in engineering applications, the control is only allowed to assume a finite number of values. Such problems are called optimal discrete-valued control problems. Optimal discretevalued control problems arise in many applications, including train control [2], switched amplifier design [11], submarine operation [10], sensor scheduling [16] and hybrid power system design $[15,13]$. To solve an optimal discrete-valued control problem, we need to determine the order in which the different control values are operated, as well as the times at which the control switches from one value to another. Since the ordering of control values is a discrete variable, classical optimal control methods are not applicable to this type of problem.

In [2], the driving strategy for a diesel train traveling on a level track is considered. The train only has three modes of operation - accelerate, coast and brake - and thus the problem of controlling the train so that fuel consumption is minimized is an optimal discrete-valued control problem. An optimality condition is derived in [2] for solving this problem. However, this condition is only applicable to the train problem, and is not applicable to general optimal discrete-valued control problems.

In [6], a time-scaling transformation technique is developed for solving optimal discrete-valued control problems. Under this transformation, the original problem with variable control switching points is transformed into an ordinary optimal control problem with known and fixed switching points. Thus, the transformed problem can be easily solved by many existing optimal control methods. However, the time-scaling transformation introduces many additional switches, and therefore the transformed problem is not equivalent to the original problem.

In [14], a new approach is proposed for solving nonlinear mixed discrete programming problems. The idea is to introduce a set of new continuous variables and transform the discrete programming problem into an ordinary optimization problem. In principle, this new problem can be solved using many existing nonlinear programming techniques. However, the transformation introduces additional equality and inequality constraints, and these constraints are extremely difficult to satisfy in practice.

In [17], an exact penalty method is proposed for solving semi-infinite programming problems. This method is adapted in [5] to develop an effective algorithm for solving optimal control problems with continuous inequality constraints. It is shown that, under some mild assumptions, making the penalty parameter sufficiently large forces the continuous inequality constraints to be satisfied. In this case, a local optimal solution of the penalty problem is also a local optimal solution of the original problem.

In this paper, we consider a class of optimal discrete-valued control problems, where there is an upper bound on the maximum number of control switches. We first apply the transformation reported in [14], under which the discrete-valued control is expressed as a linear combination of piecewise constant controls subject to a linear equality constraint and a set of quadratic 
inequality constraints. The original problem can then be written equivalently as an optimal control problem with piecewise constant controls subject to the original inequality constraints and the new constraints. Then, the time-scaling transformation [6] is applied to the transformed problem, yielding an optimal control problem with piecewise constant controls and fixed switching times. To solve this new problem, we introduce an exact penalty function, which is motivated by the work in [5,17-19], and construct a corresponding penalty problem. Convergence results show that when the penalty parameter is sufficiently large, the penalty problem is equivalent to the original problem. This penalty problem can be solved easily by many existing optimization software packages. Numerical results show that our approach can effectively solve two difficult train control problems.

\section{Problem Formulation}

\subsection{A Discrete-Valued Control Problem}

Consider the following dynamic system on the time horizon $[0, T]$ :

$$
\dot{\mathbf{x}}(t)=\mathbf{f}(\mathbf{x}(t), \mathbf{u}(t))
$$

with the initial and terminal conditions

$$
\mathbf{x}(0)=\mathbf{x}^{0}, \quad \mathbf{x}(T)=\mathbf{x}^{f},
$$

where $\mathbf{x} \in \mathbb{R}^{n}$ is the state vector, $T$ is a given terminal time, and $\mathbf{x}^{0}$ and $\mathbf{x}^{f}$ are given vectors. We assume that the function $\mathbf{f}: \mathbb{R}^{n} \times \mathbb{R}^{r} \rightarrow \mathbb{R}^{n}$ is continuously differentiable with respect to each of its arguments.

Let

$$
\mathbf{U}=\left\{\mathbf{u}_{1}, \mathbf{u}_{2}, \cdots, \mathbf{u}_{m}\right\},
$$

where each $\mathbf{u}_{j} \in \mathbb{R}^{r}$ is a given vector. We assume that the control $\mathbf{u}$ is a discrete-valued control taking values in $\mathbf{U}$. Thus, $\mathbf{u}$ is completely determined by specifying:

- The order in which it assumes the different values in $\mathbf{U}$ (the so-called switching sequence); and

- The times at which it switches from one value in $\mathbf{U}$ to another (the so-called switching times).

In this paper, we assume that there is an upper bound $N$ on the maximum number of control switches. A function $\mathbf{u}:[0, T] \rightarrow \mathbf{U}$ with at most $N$ switches/discontinuities is called an admissible control. Let $\mathscr{U}$ denote the class of all such admissible controls.

Our optimal discrete-valued control problem is stated as follows: Given the dynamic system (1)-(2), find an admissible control $\mathbf{u} \in \mathscr{U}$ such that the cost function

$$
J(\mathbf{u})=\int_{0}^{T} \mathscr{L}_{0}(\mathbf{x}(t), \mathbf{u}(t)) d t
$$


is minimized subject to the constraints

$$
g_{i}(\mathbf{x}(t), \mathbf{u}(t)) \leq 0, \quad t \in[0, T], \quad i=1,2, \cdots, p .
$$

Let this problem be referred to as Problem $(P)$. Here, we assume that the functions $\mathscr{L}_{0}$ and $g_{i}, i=1, \cdots, p$, are continuously differentiable with respect to each of their arguments.

Most numerical techniques for solving nonlinear optimal control problems - for example, control parametrization (see [12]) and state discretization (see $[1,4])$ - are only applicable when the control range is a continuous set. Thus, such methods are not applicable to Problem $(P)$, in which the control range consists of a finite number of discrete points.

The time-scaling transform introduced in [6], which is also called the Control Parametrization Enhancing Technique (CPET), is an effective method for solving optimal discrete-valued control problems. This transformation involves expanding the number of control switches to allow for every possible switching sequence, and then mapping the switching times to fixed points in a new time horizon. This yields a new optimization problem that can be solved using standard optimization techniques, such as sequential quadratic programming with active set strategy (see [9]). However, the problem with this transformation is that it introduces many "artificial" switches, and thus the optimal control obtained may have more than the maximum allowable number of switches. Consequently, the transformed optimal control problem obtained by using the time-scaling transformation in [6] is not equivalent to the original problem. We will introduce an equivalent transformation in the next section.

\subsection{Problem Transformation}

Let $\mathscr{V}$ denote the class of all piecewise constant functions mapping $[0, T]$ into $\mathbb{R}^{m}$ and having no more than $N$ switches/discontinuities. Let $\mathbf{v} \in \mathscr{V}$, where $\mathbf{v}(t)=\left(v_{1}(t), v_{2}(t), \cdots, v_{m}(t)\right)$, be an auxiliary control function.

We impose the following constraints:

$$
\begin{gathered}
\sum_{j=1}^{m} v_{j}(t)=1, \quad t \in[0, T], \\
v_{j}(t)\left(1-v_{j}(t)\right) \leq 0, \quad t \in[0, T], \quad j=1,2, \cdots, m, \\
0 \leq v_{j}(t) \leq 1, \quad t \in[0, T], \quad j=1,2, \cdots, m .
\end{gathered}
$$

The constraints (5) ensure that at each time $t \in[0, T]$, there exists exactly one $j \in\{1, \cdots, m\}$ such that $v_{j}(t)=1$ and $v_{k}(t)=0$ for all $k \neq j$.

To continue, we let

$$
\overline{\mathbf{u}}(t)=\sum_{j=1}^{m} v_{j}(t) \mathbf{u}_{j} .
$$


Since $\mathbf{v} \in \mathscr{V}$ and constraints (5) hold, $\overline{\mathbf{u}}(t) \in \mathbf{U}$ for all $t \in[0, T]$. Moreover, since $\mathbf{v}$ contains at most $N$ switches, so does $\overline{\mathbf{u}}$. It follows that $\overline{\mathbf{u}}$ is an admissible control for Problem $(P)$. In fact, it is easy to see that any admissible control for Problem $(P)$ can be written in the form of (6). Thus, by substituting $\mathbf{u}(t)=\overline{\mathbf{u}}(t)$ into the dynamical system (1), we obtain

$$
\dot{\mathbf{x}}(t)=\sum_{j=1}^{m} v_{j}(t) \mathbf{f}\left(\mathbf{x}(t), \mathbf{u}_{j}\right)
$$

Similarly, the constraints (4) become

$$
\sum_{j=1}^{m} v_{j}(t) g_{i}\left(\mathbf{x}(t), \mathbf{u}_{j}\right) \leq 0, \quad t \in[0, T], \quad i=1,2, \cdots, p .
$$

Our new optimal control problem is stated as follows: Given the dynamic system (7) with the initial and terminal conditions (2), find a control $\mathbf{v} \in \mathscr{V}$ such that the cost function

$$
\bar{J}(\mathbf{v})=J(\overline{\mathbf{u}})=\sum_{j=1}^{m} \int_{0}^{T} v_{j}(t) \mathscr{L}_{0}\left(\mathbf{x}(t), \mathbf{u}_{j}\right) d t
$$

is minimized subject to constraints (5) and (8). Let this problem be referred to as Problem $(\bar{P})$.

It is clear that Problems $(\bar{P})$ and $(P)$ are equivalent. Thus, we have the following result.

Theorem 1 Let $\mathbf{v}^{\star}=\left(v_{1}^{\star}, v_{2}^{\star}, \cdots, v_{m}^{\star}\right) \in \mathscr{V}$ and

$$
\overline{\mathbf{u}}^{\star}(t)=\sum_{j=1}^{m} v_{j}^{\star}(t) \mathbf{u}_{j} .
$$

Then $\mathbf{v}^{\star}$ is an optimal control for Problem $(\bar{P})$ if and only if $\overline{\mathbf{u}}^{\star}$ is an optimal control for Problem $(P)$.

Problem $(\bar{P})$ is a standard optimal control problem subject to the continuous inequality constraints (8) and the new constraints (5). In principle, many optimal control software packages - for example, MISER [3] — can be used to solve this problem. However, in reality, there are two major difficulties that prevent us from solving Problem $(\bar{P})$ directly:

- The switching times for the new controls $v_{j}$ are decision variables.

- The feasible region defined by the constraints (5) is a disconnected set.

We can overcome the first difficulty by applying the time-scaling transformation (see [6]), in which the variable switching times are mapped into fixed switching times. For the second difficulty, we will introduce an exact penalty function method that is inspired by our previous work in [5]. The details are provided in the next section. 


\section{Solution Procedure}

\subsection{Time-Scaling Transformation}

Recall that the control $\mathbf{v} \in \mathscr{V}$ in Problem $(\bar{P})$ has at most $N$ switches. Let $\tau_{k}$ denote the $k$ th switching time. Then

$$
0=\tau_{0} \leq \tau_{1} \leq \tau_{2} \leq \cdots \leq \tau_{N+1}=T
$$

We map these switching times to fixed time points as follows. Let $s \in[0, N+1]$ be a new time variable, and relate $t$ to $s$ by the following differential equation:

$$
\begin{aligned}
& \dot{t}(s)=\mu(s), \\
& t(0)=0,
\end{aligned}
$$

where $\mu(s)=\theta_{k}=\tau_{k}-\tau_{k-1}$ for $s \in[k-1, k), k=1, \cdots, N+1$. We can express the piecewise constant function $\mu$ as follows:

$$
\mu(s)=\sum_{k=1}^{N+1} \theta_{k} \chi_{[k-1, k)}(s),
$$

where $\chi_{I}$ is the indicator function of $I$ defined by

$$
\chi_{I}(s)= \begin{cases}1, & \text { if } s \in I \\ 0, & \text { otherwise }\end{cases}
$$

Let $\boldsymbol{\theta}=\left[\theta_{1}, \cdots, \theta_{N+1}\right]^{T} \in \mathbb{R}^{N+1}$, and note that $\theta_{k}=\tau_{k}-\tau_{k-1}$ is the duration of the $k$ th control value. For each $k=1, \cdots, N+1$, we have

$$
\begin{aligned}
t(k) & =\int_{0}^{k} \mu(s) d s \\
& =\int_{0}^{k}\left[\theta_{1} \chi_{[0,1)}(s)+\cdots+\theta_{N+1} \chi_{[N, N+1]}(s)\right] d s \\
& =\theta_{1}+\cdots+\theta_{k}=\tau_{k} .
\end{aligned}
$$

This shows that the transformation (9) maps each integer $k$ to the $k$ th switching time. Furthermore,

$$
t(N+1)=\int_{0}^{N+1} \mu(s) d s=\sum_{l=1}^{N+1} \theta_{l}=T .
$$

Clearly,

$$
0 \leq \theta_{k}=\tau_{k}-\tau_{k-1} \leq T, \quad k=1, \cdots, N+1
$$

Thus,

$$
0 \leq \mu(s) \leq T, \quad s \in[0, N+1] .
$$


Under the time-scaling transform, the control $v_{j}$ in Problem $(\bar{P})$ becomes

$$
\tilde{v}_{j}(s)=v_{j}(t(s))=\sum_{k=1}^{N+1} \xi_{j k} \chi_{[k-1, k)}(s),
$$

where $\xi_{j k}$ is the value of $v_{j}$ on $\left[\tau_{k-1}, \tau_{k}\right)$. Constraints (5) become:

$$
\begin{gathered}
\sum_{j=1}^{m} \xi_{j k}=1, \quad k=1, \cdots, N+1, \\
\xi_{j k}\left(1-\xi_{j k}\right) \leq 0, \quad j=1, \cdots, m, \quad k=1, \cdots, N+1, \\
0 \leq \xi_{j k} \leq 1, \quad j=1, \cdots, m, \quad k=1, \cdots, N+1 .
\end{gathered}
$$

Define

$$
\boldsymbol{\xi}_{j}=\left[\xi_{j 1}, \cdots, \xi_{j(N+1)}\right]^{T} \in \mathbb{R}^{N+1}
$$

and

$$
\boldsymbol{\xi}=\left[\boldsymbol{\xi}_{1}^{T}, \cdots, \boldsymbol{\xi}_{m}^{T}\right]^{T} \in \mathbb{R}^{m \times(N+1)} .
$$

Now, by applying the time-scaling transform to Problem $(\bar{P})$, the dynamical system (7) becomes

$$
\frac{d \tilde{\mathbf{x}}(s)}{d s}=\mu(s) \sum_{j=1}^{m} \tilde{v}_{j}(s) \mathbf{f}\left(\tilde{\mathbf{x}}(s), \mathbf{u}_{j}\right)=\sum_{k=1}^{N+1} \sum_{j=1}^{m} \theta_{k} \xi_{j k} \mathbf{f}\left(\tilde{\mathbf{x}}(s), \mathbf{u}_{j}\right) \chi_{[k-1, k)}(s),
$$

where

$$
\tilde{\mathbf{x}}(s)=\mathbf{x}(t(s))
$$

The initial and terminal conditions (2) become

$$
\tilde{\mathbf{x}}(0)=\mathbf{x}^{0}, \quad \tilde{\mathbf{x}}(N+1)=\mathbf{x}^{f} .
$$

Problem $(\bar{P})$ may now be written equivalently as the following problem, which we call Problem $(\tilde{P})$ : Given the dynamic system (13)-(14), find $\boldsymbol{\theta} \in \mathbb{R}^{N+1}$ and $\boldsymbol{\xi} \in \mathbb{R}^{m \times(N+1)}$ such that the cost function

$$
\tilde{J}(\boldsymbol{\theta}, \boldsymbol{\xi})=\int_{0}^{N+1} \tilde{\mathscr{L}}_{0}(s, \tilde{\mathbf{x}}(s), \boldsymbol{\theta}, \boldsymbol{\xi}) d s
$$

where

$$
\tilde{\mathscr{L}}_{0}(s, \tilde{\mathbf{x}}(s), \boldsymbol{\theta}, \boldsymbol{\xi})=\sum_{k=1}^{N+1} \sum_{j=1}^{m} \theta_{k} \xi_{j k} \mathscr{L}_{0}\left(\tilde{\mathbf{x}}(s), \mathbf{u}_{j}\right) \chi_{[k-1, k)}(s),
$$

is minimized subject to the constraints

$$
\begin{aligned}
& \tilde{g}_{i}(s, \tilde{\mathbf{x}}(s), \boldsymbol{\xi})=\sum_{k=1}^{N+1} \sum_{j=1}^{m} \xi_{j k} g_{i}\left(\tilde{\mathbf{x}}(s), \mathbf{u}_{j}\right) \chi_{[k-1, k)}(s) \leq 0, \\
& s \in[0, N+1], \quad i=1, \cdots, p,
\end{aligned}
$$

and constraints (10), (11) as well as (12).

In the next section, we will introduce an exact penalty function to develop an effective computational method for solving Problem $(\tilde{P})$. 


\subsection{An Exact Penalty Function}

Problem $(\tilde{P})$ is an optimal control problem subject to the linear constraints (10), (12a) and (12c), the quadratic constraints (12b), and the nonlinear continuous inequality constraints (15). The continuous inequality constraints (15) are continuously differentiable with respect to each of their arguments. By adopting the idea introduced in [5], we construct the following exact penalty function:

$$
F_{\kappa}(\boldsymbol{\theta}, \boldsymbol{\xi}, \epsilon)= \begin{cases}\tilde{J}(\boldsymbol{\theta}, \boldsymbol{\xi}), & \text { if } \epsilon=0, \\ \tilde{J}(\boldsymbol{\theta}, \boldsymbol{\xi})+\epsilon^{-\alpha} \Delta(\boldsymbol{\theta}, \boldsymbol{\xi}, \epsilon)+\kappa \epsilon^{\beta}, & \text { if } \epsilon>0, \\ +\infty, & \text { otherwise }\end{cases}
$$

where $\epsilon>0$ is a new decision variable, and the constraint violation $\Delta(\boldsymbol{\theta}, \boldsymbol{\xi}, \epsilon)$ is defined by

$$
\begin{aligned}
\Delta(\boldsymbol{\theta}, \boldsymbol{\xi}, \epsilon) & =\sum_{k=1}^{N+1} \sum_{j=1}^{m} \max \left\{0, \xi_{j k}\left(1-\xi_{j k}\right)-\epsilon^{\gamma}\right\}^{2}+\sum_{k=1}^{N+1} \sum_{j=1}^{m} \max \left\{0, \xi_{j k}-1-\epsilon^{\gamma}\right\}^{2} \\
& +\sum_{k=1}^{N+1} \sum_{j=1}^{m} \max \left\{0,-\xi_{j k}-\epsilon^{\gamma}\right\}^{2}+\sum_{k=1}^{N+1}\left\{\sum_{j=1}^{m} \xi_{j k}-1-\epsilon^{\gamma}\right\}^{2} \\
& +\sum_{i=1}^{p} \int_{0}^{N+1} \max \left\{0, \tilde{g}_{i}(s, \tilde{\mathbf{x}}(s), \boldsymbol{\xi})-\epsilon^{\gamma}\right\}^{2} d s+\left(t(N+1)-T-\epsilon^{\gamma}\right)^{2} \\
& +\sum_{k=1}^{N+1} \max \left\{0,-\theta_{k}-\epsilon^{\gamma}\right\}^{2} .
\end{aligned}
$$

Here, $\alpha, \beta$ and $\gamma$ are positive real numbers, and $\kappa$ is a penalty parameter. Next, we define

$$
\begin{aligned}
& S_{\epsilon}=\left\{(\boldsymbol{\theta}, \boldsymbol{\xi}, \epsilon) \in \mathbb{R}^{N+1} \times \mathbb{R}^{m \times(N+1)} \times[0, \infty):\right. \\
& t(N+1)-T=\epsilon^{\gamma} \\
&-\theta_{k} \leq \epsilon^{\gamma}, \quad k=1, \cdots, N+1, \\
& \sum_{j=1}^{m} \xi_{j k}-1=\epsilon^{\gamma}, \quad k=1, \cdots, N+1, \\
& \xi_{j k}\left(1-\xi_{j k}\right) \leq \epsilon^{\gamma}, \quad j=1, \cdots, m, \quad k=1, \cdots, N+1, \\
& \xi_{j k}-1 \leq \epsilon^{\gamma}, \quad j=1, \cdots, m, \quad k=1, \cdots, N+1, \\
&-\xi_{j k} \leq \epsilon^{\gamma}, \quad j=1, \cdots, m, \quad k=1, \cdots, N+1, \\
&\left.\sum_{k=1}^{N+1} \sum_{j=1}^{m} \xi_{j k} g_{i}\left(\tilde{\mathbf{x}}(s), \mathbf{u}_{j}\right) \chi_{[k-1, k)}(s) \leq \epsilon^{\gamma}, \quad i=1,2, \cdots, p\right\} .
\end{aligned}
$$


Now, consider the following problem: Given the dynamical system (13)-(14), find a triple $(\boldsymbol{\theta}, \boldsymbol{\xi}, \epsilon) \in \mathbb{R}^{N+1} \times \mathbb{R}^{m \times(N+1)} \times[0, \infty)$ such that the penalty function $F_{\kappa}(\boldsymbol{\theta}, \boldsymbol{\xi}, \epsilon)$ is minimized. This problem is referred to as Problem $\left(\tilde{P}_{\kappa}\right)$.

In the next section, we will see that, under some mild assumptions, when the penalty parameter $\kappa$ is sufficiently large, the satisfaction of the constraints (10), (11), (12) and (15) will be achieved, i.e. $\Delta(\boldsymbol{\theta}, \boldsymbol{\xi}, \epsilon)=0$ for $\epsilon=0$. Furthermore, an optimal solution of Problem $\left(\tilde{P}_{\kappa}\right)$ is an optimal solution of Problem $(\tilde{P})$.

\subsection{Convergence Results}

To obtain our main result, we need the following definition.

Definition 1 Suppose that the following implication holds:

$$
\sum_{i=1}^{p} \int_{0}^{N+1} \varphi_{i}(s) \frac{\partial \tilde{g}_{i}(s, \tilde{\mathbf{x}}(s), \boldsymbol{\xi})}{\partial \boldsymbol{\xi}} d s=0 \Longrightarrow \varphi_{i}(s)=0
$$

for all $s \in[0, N+1], i=1, \cdots, p$. Then, we say that the constraint qualification is satisfied for the continuous inequality constraints (15).

Let $\left\{\kappa_{l}\right\}_{l=1}^{\infty}$ be an increasing sequence of penalty parameters such that $\kappa_{l} \rightarrow \infty$. Furthermore, let $\left(\boldsymbol{\theta}^{(l), *}, \boldsymbol{\xi}^{(l), *}, \epsilon^{(l), *}\right)$ denote a local optimal solution of Problem $\left(\tilde{P}_{\kappa_{l}}\right)$. We assume that the following hypotheses are satisfied.

$\left(H_{1}\right)$ The constraint qualification defined in Definition 1 is satisfied at $(\boldsymbol{\theta}, \boldsymbol{\xi})=$ $\left(\boldsymbol{\theta}^{*}, \boldsymbol{\xi}^{*}\right)$, where $\left(\boldsymbol{\theta}^{*}, \boldsymbol{\xi}^{*}\right)$ is a local optimal solution of Problem $(\tilde{P})$.

$\left(H_{2}\right)$ There exists real numbers $\delta^{1}>0$ and $\delta^{2}>0$ such that

$$
\lim _{l \rightarrow \infty} \frac{\max \left\{0, G_{\iota}\left(s, \tilde{\mathbf{x}}(s), \boldsymbol{\theta}^{(l), *}, \boldsymbol{\xi}^{(l), *}\right)\right\}}{\left(\epsilon^{(l), *}\right)^{\delta^{1}}}=0
$$

and

$$
\lim _{l \rightarrow \infty} \frac{H_{\eta}\left(s, \tilde{\mathbf{x}}(s), \boldsymbol{\theta}^{(l), *}, \boldsymbol{\xi}^{(l), *}\right)}{\left(\epsilon^{(l), *}\right)^{\delta^{2}}}=0
$$

where $G_{\iota}, \iota=1, \cdots, p+(3 m+1)(N+1)$ and $H_{\eta}, \eta=1, \cdots, N+2$, are, respectively, the inequality constraints and the equality constraints of Problem $(\tilde{P})$.

Theorem 2 Suppose that $\left(\boldsymbol{\theta}^{(l), *}, \boldsymbol{\xi}^{(l), *}, \epsilon^{(l), *}\right) \rightarrow\left(\boldsymbol{\theta}^{*}, \boldsymbol{\xi}^{*}, \epsilon^{*}\right)$ as $l \rightarrow+\infty$, and that the hypotheses $\left(H_{1}\right)-\left(H_{2}\right)$ are satisfied. Then, $\epsilon^{*}=0$ and $\left(\boldsymbol{\theta}^{*}, \boldsymbol{\xi}^{*}\right) \in S_{0}$, where $S_{0}$ is defined by (16) with $\epsilon=0$. 
Theorem 3 Suppose that $\gamma>\alpha, \delta=\min \left(\delta^{1}, \delta^{2}\right)>\alpha, 2 \delta>\alpha+1,2 \gamma>\alpha+1$. Then

$$
\begin{gathered}
F_{\kappa_{l}}\left(\boldsymbol{\theta}^{(l), *}, \boldsymbol{\xi}^{(l), *}, \epsilon^{(l), *}\right) \underset{\left(\boldsymbol{\theta}^{(l), *}, \boldsymbol{\xi}^{(l), *}\right) \rightarrow\left(\boldsymbol{\theta}^{*}, \boldsymbol{\xi}^{*}\right) \in S_{0}}{\longrightarrow} F_{\kappa_{l}}\left(\boldsymbol{\theta}^{*}, \boldsymbol{\xi}^{*}, 0\right)=\tilde{J}\left(\boldsymbol{\theta}^{*}, \boldsymbol{\xi}^{*}\right) \\
\nabla F_{\kappa_{l}}\left(\boldsymbol{\theta}^{(l), *}, \boldsymbol{\xi}^{(l), *}, \epsilon^{(l), *}\right) \underset{\left(\boldsymbol{\theta}^{(l), *}, \boldsymbol{\xi}^{(l), *}\right) \rightarrow\left(\boldsymbol{\theta}^{*}, \boldsymbol{\xi}^{*}\right) \in S_{0}}{\longrightarrow} \nabla F_{\kappa_{l}}\left(\boldsymbol{\theta}^{*}, \boldsymbol{\xi}^{*}, 0\right)=\left(\nabla \tilde{J}\left(\boldsymbol{\theta}^{*}, \boldsymbol{\xi}^{*}\right), 0\right) .
\end{gathered}
$$

Theorem 4 Let $\epsilon^{(l), *} \rightarrow \epsilon^{*}=0,\left(\boldsymbol{\theta}^{(l), *}, \boldsymbol{\xi}^{(l), *}\right) \rightarrow\left(\boldsymbol{\theta}^{*}, \boldsymbol{\xi}^{*}\right) \in S_{0}$ be such that $F_{\kappa_{l}}\left(\boldsymbol{\theta}^{*}, \boldsymbol{\xi}^{*}, \epsilon^{*}\right)$ is finite, where $S_{0}$ is as defined by (16) with $\epsilon=0$. Then, $\left(\boldsymbol{\theta}^{*}, \boldsymbol{\xi}^{*}\right)$ is a local optimal solution of Problem $(\tilde{P})$.

Theorem 5 Suppose that $\left(\boldsymbol{\theta}^{(l), *}, \boldsymbol{\xi}^{(l), *}, \epsilon^{(l), *}\right) \rightarrow\left(\boldsymbol{\theta}^{*}, \boldsymbol{\xi}^{*}, \epsilon^{*}\right)$ as $l \rightarrow+\infty$, and that the parameters $\alpha$ and $\gamma$ satisfy the same conditions as in Theorem 3. Then, there exists a $l_{0}>0$ such that $\epsilon^{(l), *}=0$ and $\left(\boldsymbol{\theta}^{(l), *}, \boldsymbol{\xi}^{(l), *}\right)=\left(\boldsymbol{\theta}^{*}, \boldsymbol{\xi}_{\tilde{P}}^{*}\right)$, for all $l \geq l_{0}$. Furthermore $\left(\boldsymbol{\theta}^{*}, \boldsymbol{\xi}^{*}\right)$ is a local optimal solution of Problem $(\tilde{P})$.

The proofs of these theorems are quite similar to the proofs of Theorems 5.15.4 in [5] and Theorem 4 in [8]. Thus, they are omitted. From the results above, we can conclude that under some mild assumptions, for a sufficiently large $\kappa$, a local optimal solution of Problem $\left(\tilde{P}_{\kappa}\right)$ is a local optimal solution of Problem $(\tilde{P})$. Such a solution can then be used to construct a corresponding local solution of Problem $(P)$.

Problem $\left(\tilde{P}_{\kappa}\right)$ is a standard optimal control problem with fixed switching points and can be readily solved by various existing optimal control techniques. Here, the optimal control software package MISER 3.3 [3] is used. In the next section, two practical problems concerning optimal driving strategies for trains are solved by the proposed method.

\section{Numerical Results}

\subsection{Optimal Train Control on a Level Track}

The following model for the motion of a train is given in references $[2,6]$ :

$$
\begin{aligned}
& \dot{x}_{1}=x_{2} \\
& \dot{x}_{2}=\varphi\left(x_{2}\right) u_{1}+\zeta_{2} u_{2}+\rho\left(x_{2}\right),
\end{aligned}
$$

where $x_{1}$ is the train's distance along the track, $x_{2}$ is the train's speed, $u_{1}$ is the fuel setting and $u_{2}$ models the deceleration applied to the train by the brakes. The function $\varphi$, which models the tractive effort, is defined by

$$
\varphi\left(x_{2}\right)= \begin{cases}\zeta_{1} / x_{2}, & \text { if } x_{2} \geq \zeta_{3}+\zeta_{4}, \\ \zeta_{1} / \zeta_{3}+\eta_{1}\left(x_{2}-\zeta_{3}+\zeta_{4}\right)^{2} & \text { if } \zeta_{3}-\zeta_{4} \leq x_{2}<\zeta_{3}+\zeta_{4}, \\ +\eta_{2}\left(x_{2}-\zeta_{3}+\zeta_{4}\right)^{3}, & \text { if } x_{2}<\zeta_{3}-\zeta_{4},\end{cases}
$$




\begin{tabular}{ccccccc}
\hline$\zeta_{1}$ & $\zeta_{2}$ & $\zeta_{3}$ & $\zeta_{4}$ & $\zeta_{5}$ & $\zeta_{6}$ & $\zeta_{7}$ \\
\hline 1.5 & 1 & 1.4 & 0.1 & -0.015 & -0.00003 & -0.000006 \\
\hline
\end{tabular}

Table 1 Values of $\zeta_{i}, i=1, \cdots, 7$.

where $\zeta_{1}, \zeta_{2}, \zeta_{3}$ and $\zeta_{4}$ are constants, and

$$
\eta_{1}=\zeta_{1}\left[\left(\frac{1}{\zeta_{3}+\zeta_{4}}-\frac{1}{\zeta_{3}}\right) \frac{3}{4 \zeta_{4}^{2}}+\frac{1}{2 \zeta_{4}\left(\zeta_{3}+\zeta_{4}\right)^{2}}\right]
$$

and

$$
\eta_{2}=\zeta_{1}\left[-\left(\frac{1}{\zeta_{3}+\zeta_{4}}-\frac{1}{\zeta_{3}}\right) \frac{3}{4 \zeta_{4}^{3}}-\frac{1}{4 \zeta_{4}^{2}\left(\zeta_{3}+\zeta_{4}\right)^{2}}\right] .
$$

The function $\rho$, which models the resistive deceleration due to friction, is given by

$$
\rho\left(x_{2}\right)=\zeta_{5}+\zeta_{6} x_{2}+\zeta_{7} x_{2}^{2} .
$$

The constants $\zeta_{i}, i=1, \cdots, 7$, are defined in Table 1 . The initial and terminal states are

$$
\mathbf{x}(0)=[0,0]^{T}, \quad \mathbf{x}(1500)=[18000,0]^{T} .
$$

This means that the train starts from the origin at rest and comes to rest again 18,000 meters away at $t=1500$. Since the train is not allowed to go backwards, a non-negativity constraint is imposed on the speed,

$$
x_{2}(t) \geq 0, \quad t \in[0,1500] .
$$

The train driver can choose from three operation modes for the train: accelerate (powered by the engine), coast (no power), and brake (decelerate by the brakes). These three modes correspond to the following values for $\mathbf{u}=\left[u_{1}, u_{2}\right]^{T}:[1,0]^{T},[0,0]^{T}$, and $[0,-1]^{T}$. Thus, we have

$$
\mathbf{U}=\left\{[1,0]^{T},[0,0]^{T},[0,-1]^{T}\right\} .
$$

The objective is to minimize the fuel consumption, i.e.,

$$
\min J(\mathbf{u})=\int_{0}^{1500} u_{1}(t) d t
$$

Here, we assume that the maximum number of switches is $N=2$. We apply our method in conjunction with MISER 3.3 to solve the problem.

Figure 1 and Figure 2 show the optimal trajectory of $x_{1}$ and $x_{2}$, respectively. From the figures, we see that the train accelerates for the first quarter of the journey, then coasts almost until the end. The brakes are applied briefly at the end before the train stops.

Figure 4 and Figure 5 show the optimal controls $u_{1}$ and $u_{2}$, respectively. We see that the control $u_{2}$ stays zero for almost the entire time horizon, and assumes the value -1 less than two seconds before the end.

The minimum fuel consumption is 205.06. This is slightly higher than the result of 202.67 reported in [6], which was obtained using the control 


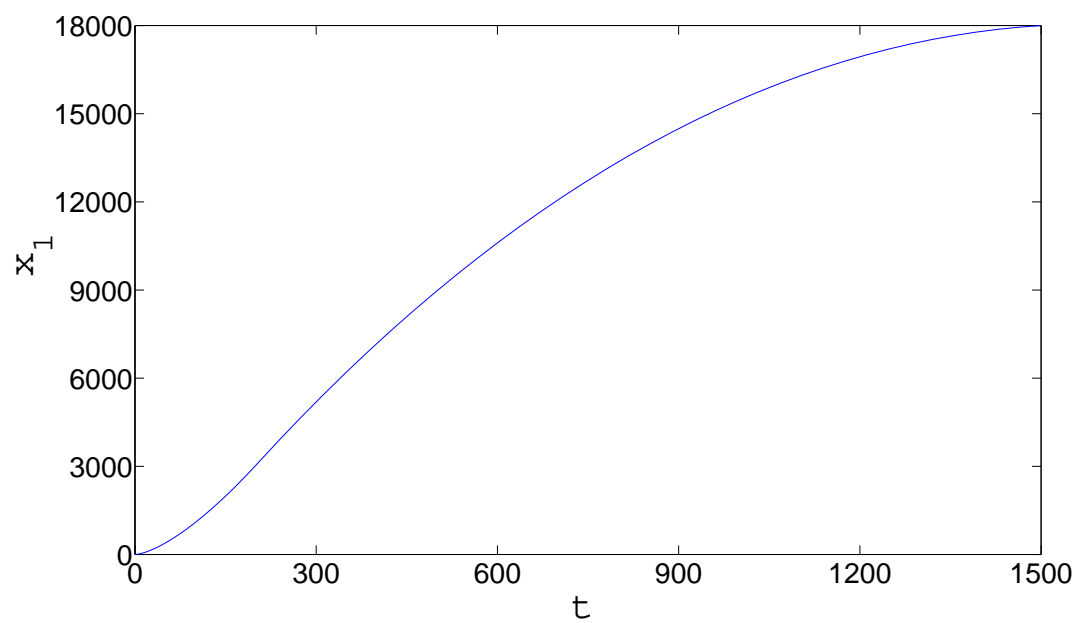

Fig. 1 The trajectory $x_{1}(t)$ against $t$

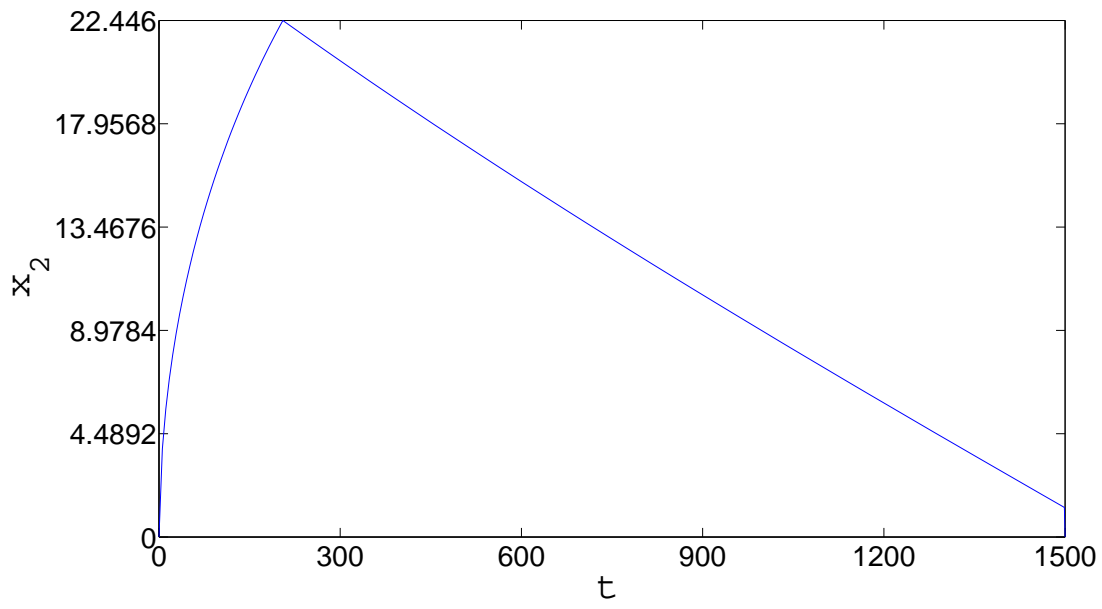

Fig. 2 The speed $x_{2}(t)$ against $t$

parametrization enhancing transform (CPET) directly with 6 switching points. It is worth noting that our method obtains the same result as in [6] when we increase the maximum number of switches to $N=6$. More importantly, unlike CPET, our method ensures that the constraint on the maximum number of switches is always satisfied. 


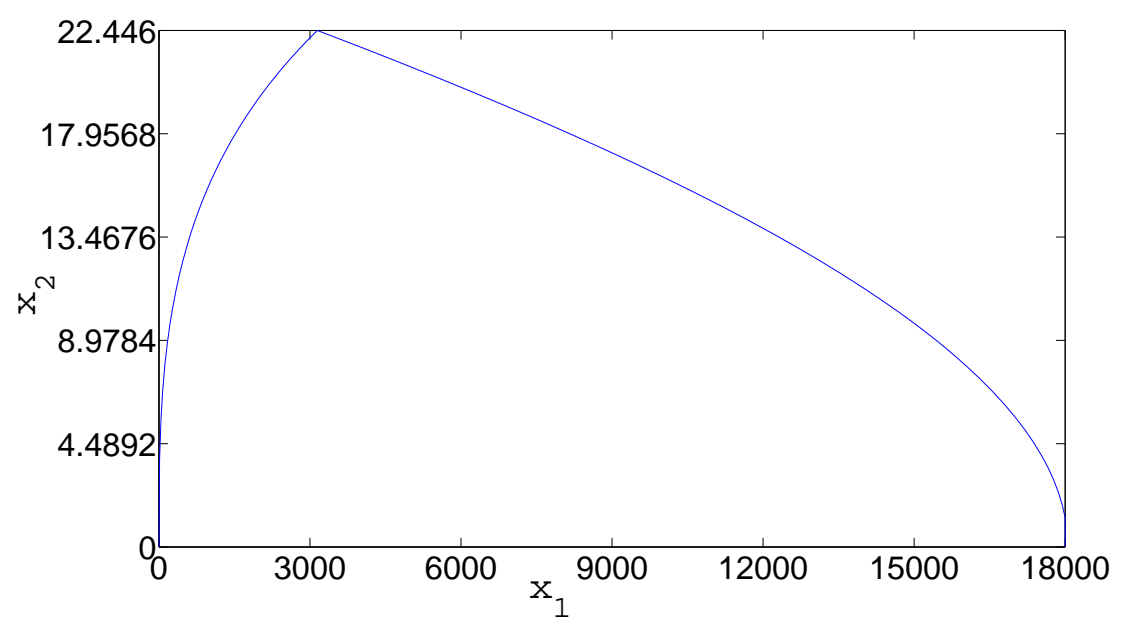

Fig. 3 The state space plot of $x_{2}$ against $x_{1}$

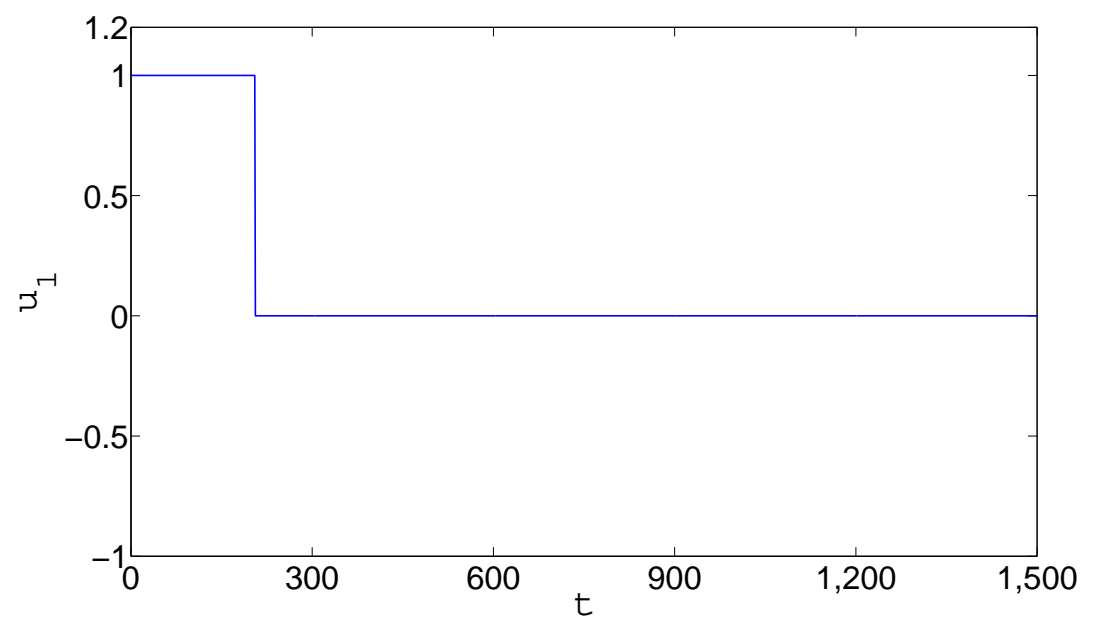

Fig. 4 The optimal control $u_{1}$ against $t$

\subsection{Optimal Train Control on an Uneven Track}

We now consider a more complicated train control problem $[2,7]$. The dynamics for this problem are

$$
\begin{aligned}
& \dot{x}_{1}=x_{2} \\
& \dot{x}_{2}=\varphi\left(x_{2}\right) u_{1}+\zeta_{2} u_{2}+\rho\left(x_{2}\right)+\vartheta\left(x_{1}\right),
\end{aligned}
$$

where $x_{1}, x_{2}, u_{1}, u_{2}, \varphi(\cdot)$ and $\rho(\cdot)$ are as defined in Example 1 , and $\xi_{i}, i=$ $1, \cdots, 7$, are as defined in Table 1 . The function $\vartheta(\cdot)$ is the gravitational ac- 


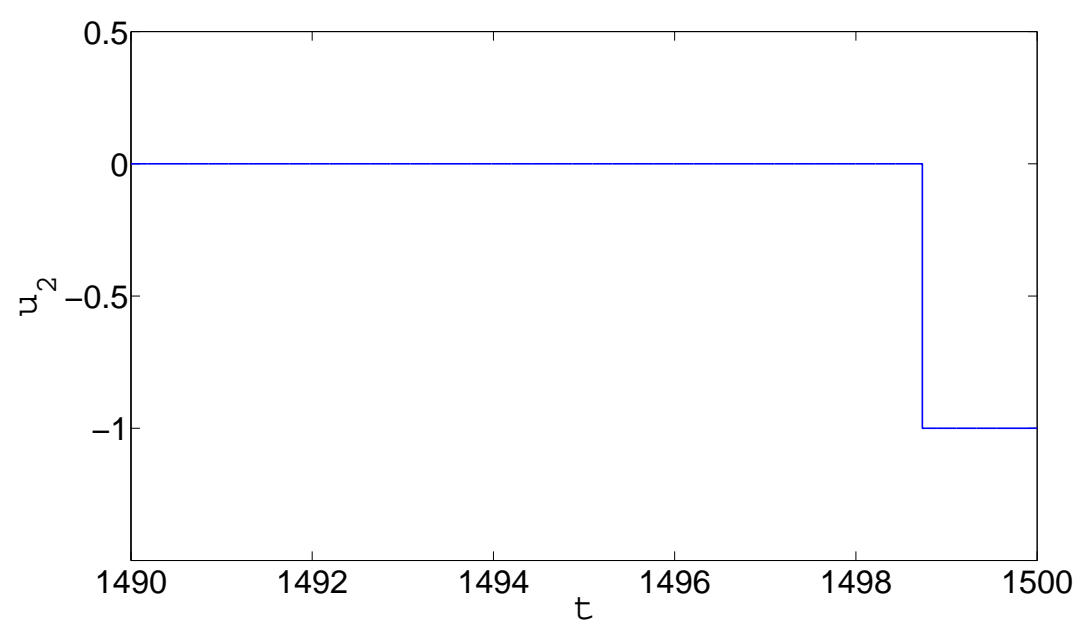

Fig. 5 The optimal control $u_{2}$ near the terminal time

celeration due to the non-constant gradient of the track given by:

$\vartheta\left(x_{1}\right)= \begin{cases}0, & \text { if } x_{1} \leq 20000-\zeta_{8}, \\ -0.05\left\{\frac{\left(x_{1}-20000\right)^{2}}{\zeta_{8}^{2}}+\frac{\left(x_{1}-2000\right)}{\zeta_{8}}+1\right\}, & \text { if } 20000-\zeta_{8}<x_{1} \leq 20000, \\ -0.05\left\{-\frac{\left(x_{1}-20000\right)^{2}}{\zeta_{8}^{2}}+\frac{\left(x_{1}-20000\right)}{\zeta_{8}}+1\right\}, & \text { if } 20000<x_{1} \leq 20000+\zeta_{8}, \\ -0.1, & \text { if } 20000+\zeta_{8}<x_{1} \leq 25000-\zeta_{8}, \\ -0.05\left\{-\frac{\left(x_{1}-25000\right)^{2}}{\zeta_{8}^{2}}-\frac{\left(x_{1}-25000\right)}{\zeta_{8}}+1\right\}, & \text { if } 25000-\zeta_{8}<x_{1} \leq 25000, \\ -0.05\left\{\frac{\left(x_{1}-25000\right)^{2}}{\zeta_{8}^{2}}-\frac{\left(x_{1}-2500\right)}{\zeta_{8}}+1\right\}, & \text { if } 25000<x_{1} \leq 25000+\zeta_{8}, \\ 0, & \text { if } x_{1}>25000+\zeta_{8},\end{cases}$

where $\zeta_{8}=300$.

The initial and terminal states are

$$
\mathbf{x}(0)=[0,0]^{T}, \quad \mathbf{x}(2800)=[50000,0]^{T} .
$$

Again, we have a non-negativity constraint on $x_{2}$ to prevent the train from going backwards:

$$
x_{2}(t) \geq 0, \quad t \in[0,2800] .
$$

We also impose a speed limit on the train that decreases as the train moves further along the track:

$$
0.0002 x_{1}(t)+x_{2}(t) \leq 28, \quad t \in[0,2800] .
$$

The control $\mathbf{u}=\left[u_{1}, u_{2}\right]^{T}$ is now restricted to the discrete set

$$
\mathbf{U}=\left\{[1,0]^{T},[0,0]^{T},[0,-1]^{T},[2,0]^{T}\right\} .
$$




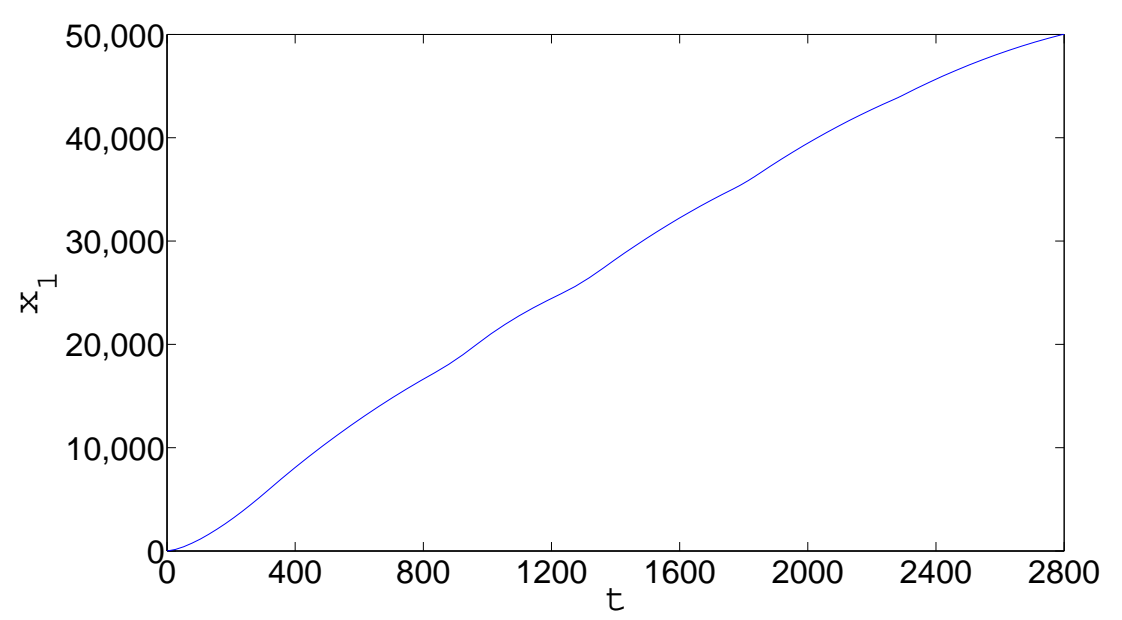

Fig. 6 The trajectory $x_{1}(t)$ against $t$

The objective is

$$
\min J(\mathbf{u})=\int_{0}^{2800} u_{1}(t) d t
$$

Here, we assume that the maximum allowable number of switches is $N=8$. Using our method, the problem is again solved by MISER 3.3. Figure 6 and Figure 7 show the optimal trajectory of $x_{1}$ and $x_{2}$, respectively. Figure 8 and Figure 9 show the optimal controls $u_{1}$ and $u_{2}$, respectively. Note that the optimal control does not assume the value $[2,0]^{T}$. From Figure 10, we can see that the continuous inequality constraint is satisfied throughout the entire time horizon.

To solve this highly complex problem, we first used our method to determine the optimal switching sequence. After identifying the optimal switching sequence, we then applied CPET directly with the control sequence fixed to refine the switching times. The minimum fuel consumption is 937.42. This is better than the result obtained in [7], which uses the CPET directly with 18 switching points and has a larger fuel consumption of 938.63.

\section{Conclusion}

In this paper, a new computational method is proposed for solving optimal discrete-valued control problems. By introducing new controls and applying an equivalent transformation, the original problem becomes a standard optimal control problem subject to equality and inequality constraints. Then, an exact penalty method is employed to solve the transformed problem. Our numerical results for the train control problems in Section 4 show that this approach is superior to the direct application of the control parametrization 


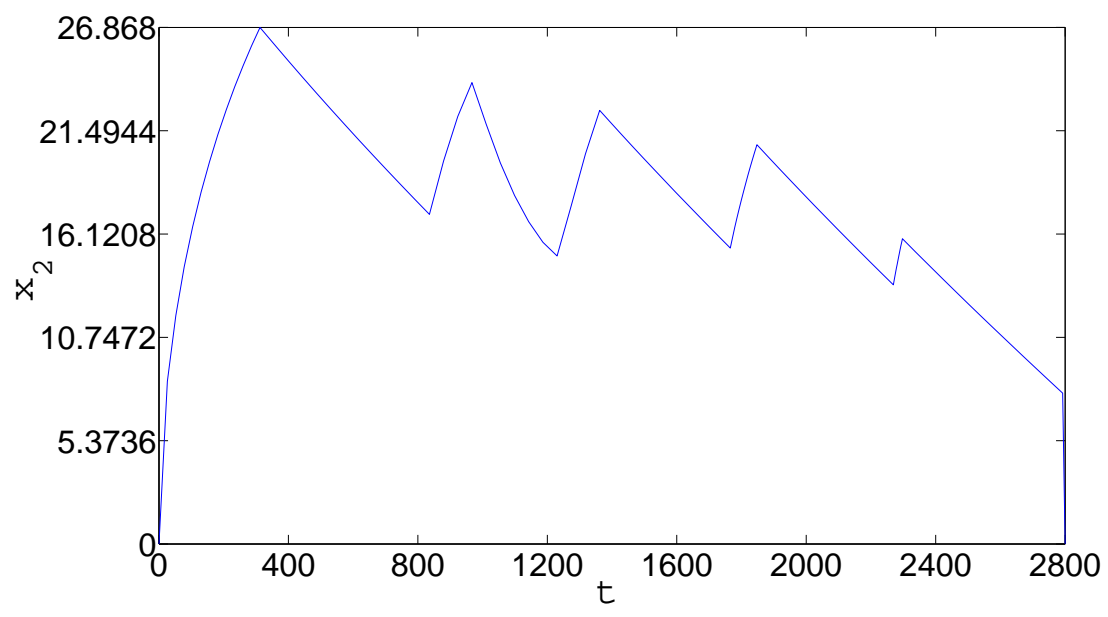

Fig. 7 The speed $x_{2}(t)$ against $t$

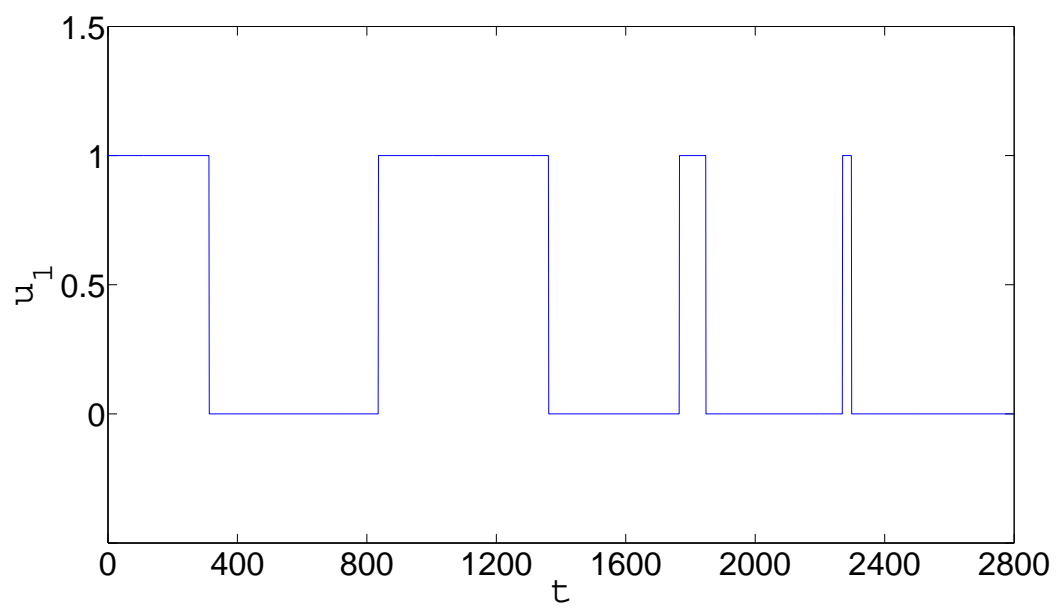

Fig. 8 The optimal control $u_{1}$ against $t$

enhancing transform, which involves introducing many artificial switches. Our optimal solutions require less switchings and always satisfy the constraint on the maximum allowable number of switchings.

Acknowledgments This work is supported by the National Natural Science Foundation of China (No. 11071158), the Key Disciplines of Shanghai Municipality (No. S30104) and the China Scholarship Council. 


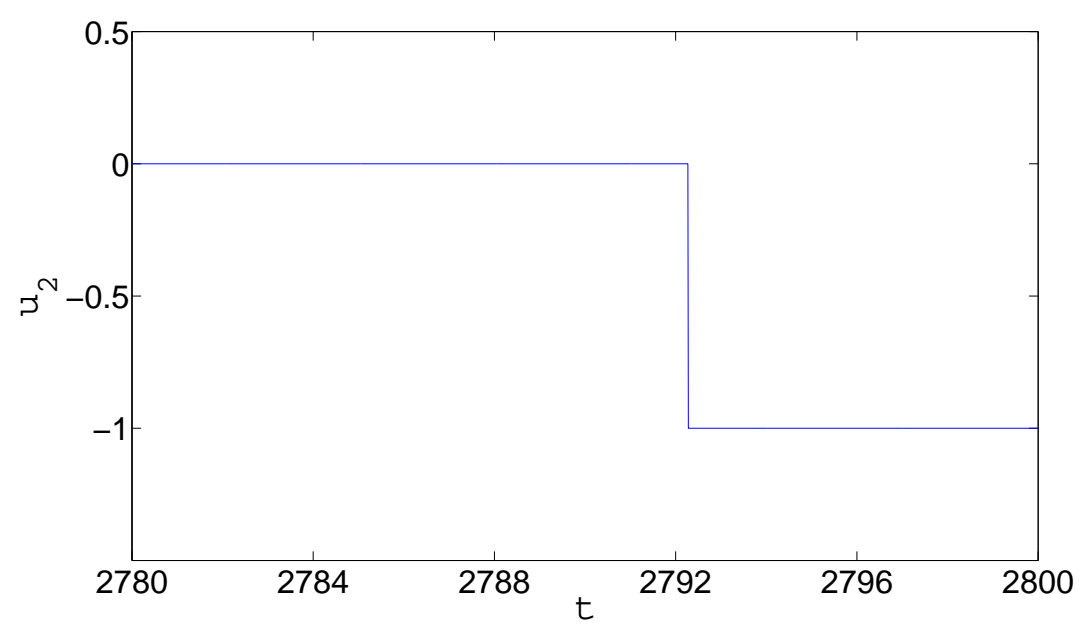

Fig. 9 The optimal control $u_{2}$ near the terminal time

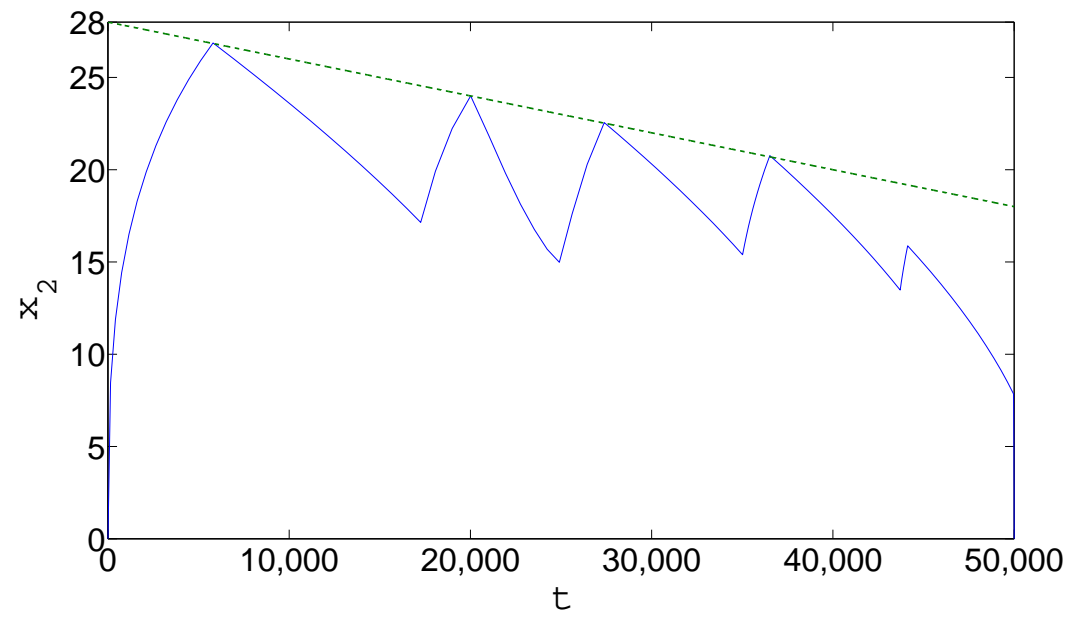

Fig. 10 The plot of speed limit constraint

\section{References}

1. Hager, W.W.: Runge-Kutta methods in optimal control and the transformed adjoint system. Numerische Mathematik. 87(2), 247-282 (2000)

2. Howlett, P.: Optimal strategies for the control of a train. Automatica. 32(4), 519-532 (1996)

3. Jennings, L.S., Fisher, M.E., Teo, K.L. and Goh, C.J.: MISER 3.3 Optimal Control Software: Theory and User Manual, University of Western Australia, Perth, Australia. (1996)

4. Kaya, C.Y. and Martínez, J.M.: Euler discretization and inexact restoration for optimal control. Journal of Optimization Theory and Applications. 134(2), 191-206 (2007). 
5. Li, B., Yu, C.J., Teo, K.L., Duan, G.R.: An exact penalty function method for continuous inequality constrained optimal control problems. Journal of Optimization Theory and Applications, DOI 10.1007/s10957-011-9904-5. (2011)

6. Lee, H.W.J., Teo, K.L., Rehbock, V. and Jennings, L.S.: Control parametrization enhancing technique for optimal discrete-valued control problems. Automatica, 35(8), 1401-1407 (1999)

7. Lee, H.W.J.: Computational studies of optimal controls, Ph.D thesis, University of Western Australia, Perth, Australia. (1996)

8. Lin, Q., Loxton, R., Teo, K.L., Wu, Y.H., Yu, C.J.: A New Exact Penalty Method for Semi-infinite Programming Problems. Submitted to Journal of Global Optimization. (2011)

9. Nocedal, J. and Wright, S.J.: Numerical Optimization, Second Edition. New York. Springer. (2006)

10. Rehbock, V., Caccetta, L.: Two defence applications involving discrete valued optimal control. ANZIAM Journal, 44, 33-54 (2002)

11. Stewart, D.E.: A numerical algorithm for optimal control problems with switching costs. Journal of the Australian Mathematical Society, Series B, 34(2) 212-228 (1996)

12. Teo, K.L., Goh, C.J. and Wong, K.H.: A unified computational approach to optimal control problems. Essex: Longman Scientific and Technical. (1991)

13. Tiryono, R., Rehbock, V., Lawrance, W.B.: Optimal control of hybrid power systems. Dynamics of Continuous, Discrete and Impulsive Systems Series B, 10(3) 429-440 (2003)

14. Wang, S., Teo, K.L., Lee, H.W.J.: A new approach to nonlinear mixed discrete programming problems. Engineering Optimization, 30(3), 249-262. (1996)

15. Woon, S.F., Rehbock, V., Loxton, R.: Towards global solutions of optimal discretevalued control problems, Optimal Control Applications and Methods, to appear

16. Woon, S.F., Rehbock, V., Loxton, R.: Global Optimization Method for ContinuousTime Sensor Scheduling, Nonlinear Dynamics and Systems Theory, 10(2), pp. 175-188, (2010)

17. Yu, C.J., Teo, K.L., Zhang, L.S., Bai, Y.Q.: A new exact penalty function method for continuous inequality constrained optimization problems. Journal of Industrial and Management Optimization, 6(4) 895-910 (2010)

18. Yu, C.J., Teo, K.L., Bai, Y.Q.: An exact penalty function method for nonlinear mixed discrete programming problems. Optimization Letters, to appear

19. Yu, C.J., Teo, K.L., Zhang, L.S., Bai, Y.Q.: On a refinement of the convergence analysis for the new exact penalty function method for continuous inequality constrained optimization problem. Journal of Industrial and Management Optimization, to appear 